\title{
Diversidade e sazonalidade de coccinelídeos (Coleoptera: Coccinellidae) em pomar no município de Ponta Grossa, Paraná
}

\author{
Bruno Piotrovski Begha ${ }^{\bowtie}$, Jana Magaly Tesserolli de Souza, Carlos Henrique Antunes \& Julianne Milléo
}

Universidade Estadual de Ponta Grossa.

\section{EntomoBrasilis 12 (3): 108-112 (2019)}

Resumo. Uma análise sobre a flutuação populacional da entomofauna associada a culturas de interesse econômico pode fornecer subsídios para o manejo integrado de pragas. Dentre os grupos economicamente relevantes podemos citar os coccinelídeos, conhecidos por serem predadores naturais de pragas agrícolas como afídeos. O presente trabalho se faz necessário visto que os Campos Gerais ainda carecem de dados sobre Coccinellidae relacionados a pomares de árvores frutíferas. O estudo foi realizado no pomar do Colégio Agrícola Estadual Augusto Ribas (Ponta Grossa-PR) de julho de 2004 a junho de 2006. As coletas foram feitas usando cada espécie do pomar, sendo elas agrupadas em dois grupos: cítricas e decíduas. Harmonia axyridis (Pallas) foi a espécie mais representativa na amostragem, compreendendo $38,35 \%$ das coletas. as árvores cítricas apresentaram o maior número de insetos totalizando $82,19 \%$ da amostragem. É pertinente sugerir que a presença da $H$. axyridis pode ter afetado a diversidade da comunidade local, sendo ela uma espécie invasora e considerada uma melhor competidora. As árvores cítricas se apresentaram como as mais diversas. Podemos atribuir essa distribuição a maior presença de afídeos nas árvores cítricas, que foram atraídos pelos óleos voláteis secretados por essas plantas. No período final da amostragem houve uma queda populacional, que pode ser atribuída a condições climáticas desfavoráveis, e consequente redução a abundância dos afídeos presa e dos próprios coccinelídeos. Uma maior quantidade de recursos de presa permitiria que mais espécies de joaninhas coexistissem sem competir fortemente. Uma pesquisa futura poderia comparar situação da comunidade, analisando a influência da $H$. axyridis nas espécies locais.

Palavras-chave: Bioecologia; controle biológico; flutuação populacional.

\section{Diversity and seasonality of coccinellids (Coleoptera: Coccinellidae) in an orchard in the city of Ponta Grossa, Paraná}

\begin{abstract}
An analysis of the population fluctuation of entomofauna associated with crops of economic interest can provide subsidies for integrated pest management. Among the economically relevant groups we can mention the Coccinellidae, known to include natural predators of agricultural pests such as aphids. The present work is necessary since Parana's Campos Gerais still lack data on Coccinellidae related to orchards of fruit trees. The study was conducted in the orchard of Augusto Ribas State Agricultural College (Ponta Grossa-PR) from July 2004 to June 2006. Samples were collected from each species of the orchard, grouped into two groups: citrus and deciduous. Harmonia axyridis (Pallas) was the most representative species in the sample, comprising $38.35 \%$ of the collections. Citrus trees had the highest number of insects totaling $82.19 \%$ of the sample. It is pertinent to suggest that the presence of $H$. axyridis may have affected the diversity of the local community, being an invasive species and considered a better competitor. The citrus trees presented themselves as the most diverse. We can attribute this contribution to the greater presence of aphids in the citrus trees, which were attracted by the volatile oils secreted by these plants. In the final period of sampling there was a decrease in population size, which can be attributed to unfavorable climatic conditions, and consequent reduction in the abundance of prey aphids and of the coccinellids themselves. A greater amount of prey resources would allow more species of ladybugs to coexist without competing strongly. A future research could compare community situation by analyzing the influence of $H$. axyridis on local species.
\end{abstract}

Keywords: Bioecology; biological control; population fluctuation.

(U) ma análise sobre a flutuação populacional da entomofauna associada a culturas de interesse econômico é de suma importância para estudos de avaliação de danos (ABreu et al. 2002) (MATRANGOlO et al. 1997), além de fornecer subsídios para o manejo integrado de pragas (ОвRускі et al. 2009). Dentre os grupos economicamente relevantes podemos citar os coccinelídeos. Coccinellidae é uma família de coleópteros cosmopolita, compreendendo
6000 espécies, divididas em 360 gêneros e 38 subfamílias (ARNETT et al. 2002). A maioria dos representantes da família são predadores, alimentando-se principalmente de afídeos, cochonilhas e ácaros. Algumas espécies são fitófagas e outras micófagas, embora seja confirmado que possam apresentar um hábito onívoro para complementar sua dieta, alimentando-se de néctar, seiva e pólen (PANIZzi \& PARRa 2009). Coccinelídeos são comumente utilizados no controle biológico conservativo
Edited by:

William Costa Rodrigues

\section{Article History:}

Received: $20 . x i i .2018$

Accepted: o9.vi.2019
Corresponding author:

Bruno Piotrovski Begha

乃bpbegha@gmail.com

(iD) No ORCID record
Funding agencies:

$\Delta$ Without funding declared 
de pragas como uma alternativa a agrotóxicos, exatamente por serem inimigos naturais de insetos fitófagos que são conhecidos como pragas agrícolas (OBRYCKI \& KRING 1998).

Partindo disso, a região dos Campos Gerais do Paraná, carece de informações a respeito da diversidade destes besouros associados a árvores frutíferas. Portanto, o presente estudo teve como objetivo principal determinar a distribuição temporal e espacial de Coccinellidae em um pomar em Ponta Grossa, Paraná; e como objetivos específicos apresentar uma análise faunística e estrutural da comunidade; avaliar a relação dos coccinelídeos com as árvores; verificar se há sazonalidade e influência climática na flutuação de coccinelídeos durante o período de amostragem.

\section{MATERIAIS E MÉTODOS}

O estudo foi realizado no pomar do Colégio Agrícola Estadual Augusto Ribas (Ponta Grossa-PR), comárea de aproximadamente $1000 \mathrm{~m}^{2}\left(25^{\circ} \mathrm{O} 5^{\prime} 42^{\prime \prime} \mathrm{S}, 50^{\circ} \mathrm{O} 6{ }^{\prime} 17\right.$ " W; $\left.900 \mathrm{~m}\right)$, de julho de 2004 a junho de 2006, totalizando 67 coletas. As coletas foram feitas quinzenalmente, no período matutino, com uso de guardachuva entomológico. Foram selecionadas previamente três árvores de cada espécie do pomar, sendo elas separadas em dois grupos: Cítricas - laranjeiras (Citrus sp.), limoeiros (Citrus sp.), tangerineiras (Citrus sp.) e, Decíduas - macieiras (Malus sp.), nectarineira (Prunus sp.), pereiras (Pyrus sp.), pessegueiros (Prunus sp.), caquizeiro (Diospyros sp.). As árvores no pomar estavam dispostas equidistantes três metros umas das outras em um terreno retangular, árvores do mesmo grupo estavam próximas umas das outras. Na área ao redor do pomar não haviam árvores ou arbustos, nem era utilizado nenhum produto fitossanitário, fatores que afetariam a distribuição dos insetos.

Nas amostragens, somente os coleópteros foram avaliados. Padronizou-se balançar por três vezes os galhos de cada uma das árvores selecionadas, provocando a queda dos insetos sobre o guarda-chuva entomológico. No laboratório, os coccinelídeos foram separados em morfoespécies, fixados em alfinetes entomológicos, etiquetados e, posteriormente, identificados ao menor táxon possível, seguindo a chave dicotômica de classificação de ARNETT et al. (2002). O material estudado encontra-se depositado na Coleção Entomológica dos Campos Gerais do Paraná (CECG), Universidade Estadual de Ponta Grossa, Ponta Grossa-PR.

Os dados foram analisados no programa ANAFAU, desenvolvido pela ESALQ (MORAES et al. 2003), no qual foram avaliados os índices de frequência, abundância, dominância e constância. De acordo com os resultados, foram selecionadas as espécies predominantes, designadas como "indicadores ecológicos" e nas quais se baseou a discussão deste trabalho. Também foram feitas análises com o software PAST versão 2.17c (HAMMER et al. 2013): a diversidade de coccinelídeos no pomar foi calculada pelos índices de Shannon-Wienner e Margalef. A uniformidade, em termos de abundância de indivíduos, distribuída entre as espécies amostradas, foi calculada pelo índice de equitatividade. Para averiguar se existia diferença significativa dentro dos dois grupos de árvores, for feita uma ANOVA unifatorial. Para a avaliação de similaridade entre as árvores frutíferas com relação à composição de espécies, foi aplicado o coeficiente de Jaccard (que utiliza dados de presença/ausência), e com relação à estrutura de comunidade, foi aplicado o índice de Morisita (que utiliza dados de abundância).

A fim de verificar a influência de fatores meteorológicos (precipitação acumulada, umidade relativa do ar, temperaturas máxima, média e mínima) na flutuação populacional ao longo da amostragem, foi realizada uma análise de correlação de Spearman entre os valores de abundância mensal total (soma de todas as plantas) e os fatores meteorológicos. Os dados climáticos foram fornecidos pelo Instituto Tecnológico SIMEPAR, Estação Meteorológica de Ponta Grossa.

\section{RESULTADOS E DISCUSSÃO}

Ao total foram coletados 764 insetos, divididos em 37 espécies. Harmonia axyridis (Pallas) foi a espécie mais representativa na amostragem, compreendendo 38,35 \% das coletas com 293 indivíduos. A segunda espécie mais representativa foi Zilini sp. 1, compreendendo 6,54\% das coletas, com 50 indivíduos (Figura 1). Todas as espécies coletadas são registradas como predadores, com exceção do gênero Psyllobora, reconhecido com micófago (SANTOS-CIVIDANEs et al. 2007).Foi possível observar que no primeiro ano completo de amostragem a população de Coccinellidae era abundante e equilibrada em termos de riqueza de espécies (477 indivíduos ao total e 36 espécies), havendo uma queda de 60,16\% no número de indivíduos e uma queda de 19,44\% no número de espécies, no segundo ano de amostragem (287 indivíduos e 29 espécies).

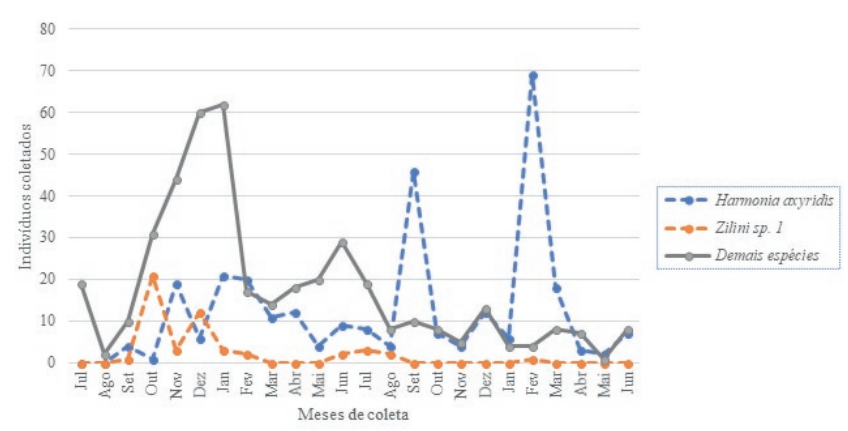

Figura 1. Flutuação populacional dos coccinelídeos coletados de julho de 2004 a junho de 2006 no pomar do Colégio Agrícola Estadual Augusto Ribas, UEPG.

Com relação às coletas nas árvores, as árvores cítricas apresentaram o maior número de insetos totalizando $82,19 \%$ da amostragem, e dentre elas as tangerineiras continham um maior número de coleópteros (Figura 2). Pela análise da ANOVA unifatorial, não se constatou diferença significativa na distribuição de espécies dentro de cada grupo de árvores.

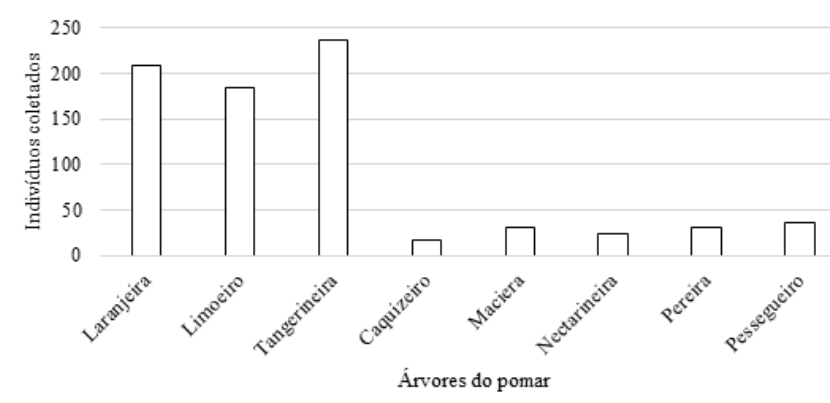

Figura 2. Distribuição dos coccinelídeos coletados de julho de 2004 a junho de 2006 nas árvores do pomar do Colégio Agrícola Estadual Augusto Ribas da UEPG.

De acordo com a análises faunísticas realizadas pelo programa ANAFAU foram contabilizadas 18 espécies dominantes em pelo menos um dos pomares (Tabela 1). As árvores cítricas apresentaram o maior número de espécies dominantes $\mathrm{e}$ constantes na amostragem.

Dentre elas, H. axyridis foi única espécie classificada como superdominante, além de ser a que se apresentou como dominante no maior número de espécies de árvore. A H. axyridis é uma joaninha nativa do leste da Ásia utilizada no controle biológico de pulgões, tendo sido introduzida na Louisiana e Mississippi, Estados Unidos, em 1990. A partir desse período 
Tabela 1. Distribuição e análise faunística das espécies dominantes de coccinelídeos coletadas no pomar do Colégio Agrícola Estadual Augusto Ribas, entre julho de 2004 a junho de 2006, no município de Ponta Grossa, PR.

\begin{tabular}{|c|c|c|c|c|c|c|c|c|}
\hline \multirow{2}{*}{ Coccinelidae } & \multicolumn{3}{|c|}{ Árvores cítricas } & \multicolumn{5}{|c|}{ Árvores decíduas } \\
\hline & Lar & Lim & Tan & Caq & Mac & Nec & Per & Pes \\
\hline Azya luteipes & - & $\mathbf{Z}$ & $\mathbf{W}$ & $\mathbf{Y}$ & - & - & $\mathbf{Y}$ & - \\
\hline Coccinelidae 2 & $\mathbf{Y}$ & $\mathbf{Y}$ & $\mathbf{Z}$ & $\mathbf{Y}$ & - & - & $\mathbf{Y}$ & - \\
\hline Curinus sp. & $\mathbf{Y}$ & $\mathbf{Z}$ & $\mathbf{W}$ & - & - & $\mathbf{Z}$ & - & - \\
\hline Cycloneda sanguinea & $\mathbf{Y}$ & $\mathbf{Y}$ & W & - & - & - & $\mathbf{Y}$ & W \\
\hline Diomini sp. 1 & - & $\mathbf{Y}$ & - & - & - & - & - & - \\
\hline Harmonia axyridis & W & W & W & W & $\mathbf{Y}$ & W & W & W \\
\hline Nephaspis ornatus & $\mathbf{Z}$ & $\mathbf{Y}$ & $\mathbf{Z}$ & - & - & - & - & - \\
\hline Olla v-nigrum & $\mathbf{Y}$ & $\mathbf{Z}$ & $\mathbf{Z}$ & - & - & - & - & - \\
\hline Pentilia egena & $\mathbf{Z}$ & $\mathbf{Y}$ & W & - & - & $\mathbf{Z}$ & - & $\mathbf{Y}$ \\
\hline Psyllobora gratiosa & $\mathbf{Z}$ & $\mathbf{Y}$ & $\mathbf{Y}$ & - & $\mathbf{Y}$ & $\mathbf{Z}$ & $\mathbf{Y}$ & $\mathbf{Z}$ \\
\hline Scymnus coniferarum & W & $\mathbf{Y}$ & $\mathbf{W}$ & $\mathbf{w}$ & - & $\mathbf{Z}$ & $\mathbf{Y}$ & - \\
\hline Scymnus loewii & w & - & $\mathbf{Z}$ & - & - & $\mathbf{w}$ & $\mathbf{Y}$ & $\mathbf{W}$ \\
\hline Scymnus sp. 1 & $\mathbf{Y}$ & - & $\mathbf{Z}$ & - & - & $\mathbf{Z}$ & - & $\mathbf{Y}$ \\
\hline Scymmus sp. 2 & $\mathbf{Y}$ & W & $\mathbf{Y}$ & $\mathbf{Y}$ & - & w & $\mathbf{Y}$ & $\mathbf{Z}$ \\
\hline Sticholotidini sp. & $\mathbf{Y}$ & W & $\mathbf{Y}$ & $\mathbf{Y}$ & $\mathbf{Y}$ & - & - & $\mathbf{Y}$ \\
\hline Zilini sp. 1 & W & $\mathbf{Z}$ & $\mathbf{w}$ & - & - & $\mathbf{Z}$ & $\mathbf{Y}$ & w \\
\hline Zilini sp. 2 & $\mathbf{Z}$ & $\mathbf{Y}$ & $\mathbf{Z}$ & $\mathbf{Y}$ & - & - & - & - \\
\hline Zilus sp. & $\mathbf{Z}$ & $\mathbf{Z}$ & W & - & - & - & - & $\mathbf{Y}$ \\
\hline
\end{tabular}

Legendas. Árvores: Laranjeira (Lar), Limoeiro (Lim), Tangerineria (Tan), Caquizeiro (Caq), Macieira (Mac), Nectarineira (Nec), Pereira (Per), Pessegueiro (Pes). Dominância: Não dominante (Amarelo) - spp cuja frequência é menor que o limite de dominância; Dominante (Laranja) - spp cuja frequência é maior que o limite de dominância; Superdominante (Vermelho). Constância (Co): Constante (W) - presente em mais de 50\% das coletas; Acessória $(\mathrm{Y})$ - presente entre 25 a 50\% das coletas; Acidental (Z) - presente em menos de $25 \%$ das coletas.

foram relatadas várias coletas com a presença desta espécie em direção à América do Sul, sendo que no Brasil sua presença foi confirmada pela primeira vez em Curitiba, Paraná, em 2002 (Almeida \& Silva 2002).

É pertinente sugerir que a presença da $H$. axyridis pode ter afetado a diversidade da comunidade local (Milléo et al. 2008). Pode-se justificar esses resultados, pelo fato dela não possuir predadores naturais na região, de seu tamanho ser maior e, as taxas de fecundidade e fertilidade mais altas em comparação com os coccinelídeos locais (Adaptado de KocH 2003). Além disso, indivíduos de $H$. axyridis possuem tendência à maior agressividade intraguilda (MICHAUD 2002).

As outras 17 espécies dominantes (Azya luteipes Mulsant, Coccinelidaesp.2,Curinussp.,Cyclonedasanguinea(Linnaeus),, Diomini sp. 1, Nephus ornatus (LeConte), Olla v-nigrum (Mulsant), Pentilia egena (Mulsant) Psyllobora gratiosa (Mader), Scymnus coniferarum (Abbot \& Smith), Scymnus loewii (Mulsant) Scymnus sp. 1, Scymnus sp. 2, Sticholotidini sp., Zilini sp. 1, Zilini sp. 2, Zilus sp.) (as espécies nativas estão destacadas em negrito) se apresentaram como dominantes em algum momento da amostragem, correspondendo a 51,04\% da coleta. Seis espécies eram exclusivas de uma espécie de árvore, porém, já que contabilizavam menos de cinco indivíduos cada, foram atribuídas como presenças acidentais.

No período final da amostragem houve uma queda populacional, que pode ser atribuída a condições climáticas desfavoráveis. Este período apresentou os menores valores para média da temperatura mínima, temperatura média, temperatura mínima e umidade relativa do ar. Tais fatores climáticos estressantes podem ter reduzido a abundância dos afídeos presa e dos próprios coccinelídeos (OLIVEIRA 1971).

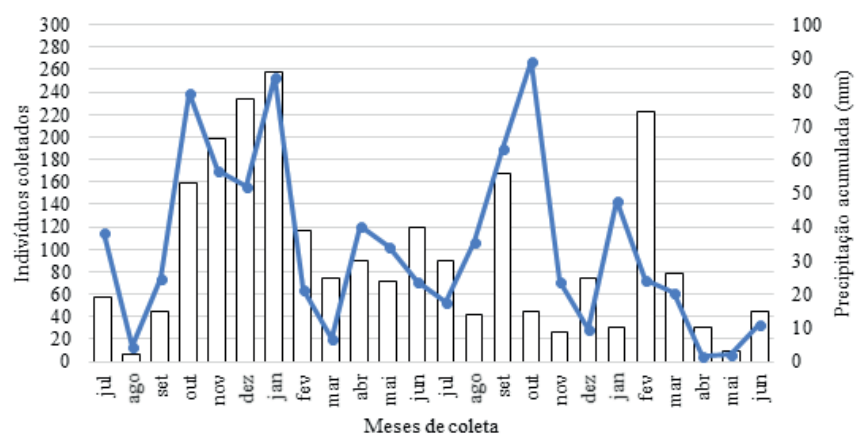

$\square$ Individuos coletados $\quad$ Precipitação Acum ulada (m m)

Figura 3. Distribuição dos coccinelídeos coletados de julho de 2004 a junho de 2006 nas árvores do pomar do Colégio Agrícola Estadual Augusto Ribas da UEPG, valores de precipitação acumulada (mm) e temperatura média $\left({ }^{\circ} \mathrm{C}\right)$.

Dendrogramas agrupando árvores frutíferas pela similaridade de fauna mostram padrões na distribuição dos insetos. Pelo índice de Jaccard (Figura 4A) observou-se um agrupamento distinto de espécies nas árvores cítricas, enquanto as árvores decíduas não apresentaram um agrupamento de destaque, com exceção da macieira, que foi a árvore que mais atraiu uma única espécie, $P$. gratiosa (10 indivíduos, sendo que as outras árvores atraíram em média apenas 2,28 indivíduos dessa espécie).

O dendrograma de similaridade obtido pelo índice de Morisita (Figura 4B) apresentou um agrupamento mais homogêneo entre a abundância presente em laranjeira, pereira, tangerineira, limoeiro; enquanto o caquizeiro, pessegueiro e nectarineira se apresentaram mais distintos em sua composição de espécies.

Foi possível observar que as árvores cítricas apresentaram uma maior diversidade de espécies ao longo de amostragem (Tabela 2). Os valores mais próximos de 1 indicam uma comunidade 


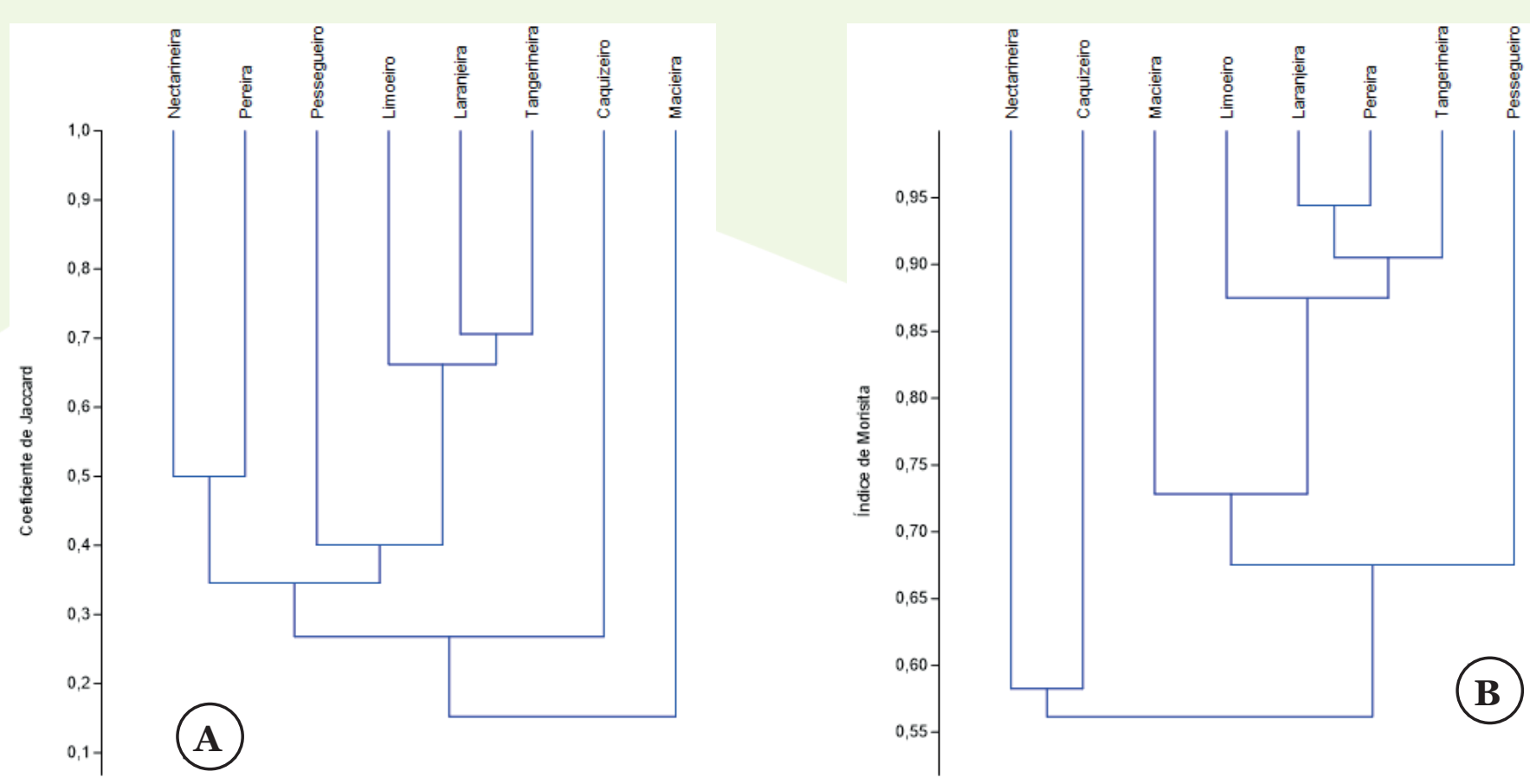

Figura 4. Dendrograma de similaridade da comunidade de Coccinellidae coletados de julho de 2004 a junho de 2006 nas árvores do pomar do Colégio Agrícola Estadual Augusto Ribas da Universidade Estadual de Ponta Grossa. A) Coeficiente de Jaccard. B) Índice de Morisita.

com poucas espécies dominantes em meio a muitas espécies raras (MAGURRAN 2003). Confirmando que nas árvores cítricas os indivíduos estavam mais bem distribuídos entre as espécies de Coccinellidae, enquanto que nas árvores decíduas haviam poucas espécies menos mais dominantes.

Tais resultados podem ser atribuídos provavelmente a maior presença de afídeos nas árvores cítricas, que por sua vez provavelmente foram atraídos pelas substâncias voláteis secretadas pelos citrinos para atrair possíveis polinizadores (RAVEN et al. 2014). A abundância de recursos de presa permitiria que várias espécies de predadores coexistissem sem competir fortemente, e sem que houvesse uma espécie limitando a existência das outras (CAIN et al. 2011).

Com relação à sazonalidade, no primeiro ano de amostragem, o verão apresentou o maior número de indivíduos e maior variedade de espécies. Durante o inverno houve uma grande queda na presença de espécies nas árvores decíduas.

De acordo com as análises de correlação de Spearman entre os fatores climáticos e a diversidade de espécies, não houve correlação significativa para a temperatura média $(\mathrm{r}=-\mathrm{O}, \mathrm{03})$
( $\mathrm{p}>0,05)$. Houve correlação significativa para a precipitação acumulada $(\mathrm{r}=0,25)(\mathrm{p}<0,05)$ e a umidade relativa do $\operatorname{ar}(\mathrm{r}=0,38)$ $(\mathrm{p}<\mathrm{0}, 05)$.

A queda da abundância geral no segundo ano de coleta pode estar relacionada a menores valores médios de umidade relativa do ar e à precipitação acumulada no segundo ciclo $(78,3 \%$ e $82,5 \mathrm{~mm}$, respectivamente) em comparação ao primeiro $(84,8 \%$ e $119,5 \mathrm{~mm})$. Tais fatores podem ter influenciado na flutuação populacional dos afídeos presa dos coccinelídeos, já que esses fitófagos estão altamente associados à fenologia de sua planta hospedeira, sendo que o período chuvoso está diretamente relacionado com o crescimento natural dos vegetais (Oliveira 1971). Após as chuvas, ocorrem novos brotamentos nas árvores, contribuindo para o aumento populacional dos afídeos e, consequentemente, para o crescimento populacional de coccinelídeos predadores. A dinâmica para coccinelídeos micófagos seria semelhante, já que a alta umidade favorece o crescimento dos fungos parasitas nas árvores (Oliveira et al. 2009).

Quando comparados com outros trabalhos (Roy \& WAJNBERG 2008) podemos observar como a dinâmica populacional da $H$. axyridis pode ter tanto um aspecto negativo de espécie invasora,

Tabela 2. Diversidade e sazonalidade de coccinelídeos no pomar do Colégio Agrícola Estadual Augusto Ribas, entre julho de 2004 a junho de 2006, no município de Ponta Grossa, PR.

\begin{tabular}{|c|c|c|c|c|c|c|c|c|c|c|c|c|c|c|c|c|}
\hline \multirow{2}{*}{\multicolumn{2}{|c|}{ Árvores }} & \multicolumn{4}{|c|}{$1^{\circ}$ ano } & \multicolumn{5}{|c|}{$2^{\circ}$ ano } & \multicolumn{6}{|c|}{ Índices } \\
\hline & & Inv & Prim & Ver & Out & Inv & Prim & Ver & Out & Inv & No & $\mathbf{S}$ & Sh & Ma & Eq & Do \\
\hline \multirow{3}{*}{ 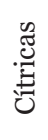 } & Laranja & 8 & 37 & 50 & 25 & 25 & 23 & 31 & 7 & 2 & 208 & 27 & 2,478 & 4,871 & 0,752 & 0,153 \\
\hline & Limão & 4 & 27 & 34 & 33 & 20 & 8 & 46 & 11 & 1 & 184 & 25 & 2,17 & 4,602 & 0,674 & 0,270 \\
\hline & Tangerina & 9 & 32 & 85 & 12 & 28 & 40 & 14 & 6 & 10 & 236 & 3 & 2,529 & 5,491 & 0,737 & 0,155 \\
\hline \multirow{5}{*}{ 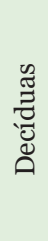 } & Caqui & o & 5 & 2 & 3 & o & 1 & o & 4 & 1 & 16 & 31 & 2,155 & 3,246 & 0,936 & 0,133 \\
\hline & Nectarina & o & 3 & 8 & 1 & 8 & 2 & 3 & 5 & o & 30 & 10 & 0,769 & 0,588 & 0,700 & 0,513 \\
\hline & Maçã & o & 4 & 11 & 2 & o & 2 & 3 & 1 & o & 23 & 12 & 2,292 & 3,508 & 0,922 & 0,119 \\
\hline & Pêra & o & 13 & 4 & 3 & 3 & 2 & 1 & 5 & o & 31 & 12 & 1,989 & 3,203 & 0,800 & 0,205 \\
\hline & Pêssego & o & 13 & 9 & o & o & 2 & 11 & o & 1 & 36 & 12 & 2,315 & 3,07 & 0,932 & 0,116 \\
\hline \multirow{2}{*}{$\stackrel{0}{2}$} & No & 21 & 134 & 203 & 79 & 84 & 80 & 109 & 39 & 15 & 764 & 37 & 2,61 & 5,423 & 0,723 & 0,168 \\
\hline & $\mathbf{S}$ & 11 & 23 & 27 & 21 & 21 & 13 & 12 & 10 & 8 & - & - & - & - & - & - \\
\hline
\end{tabular}

Po (pomar), No (número de indivíduos coletados), S (número de espécies coletadas), Sh (índice de Shannon-Wiener), Ma (índice de Margalef), Eq (equitatividade), Do (dominância). Inv (inverno), Prim (primavera), Ver (verão), Out (outubro). 
ao reduzir a quantidade de recursos para outras espécies e predá-las, quanto um aspecto positivo no controle biológico de pragas agrícolas. A partir dos dados analisados, uma pesquisa subsequente poderia comparar situação atual da comunidade, levando em conta o desenvolvimento e a influência da $H$. axyridis nas espécies locais.

Ao longo dos 29 meses de coleta, foi possível verificar que as plantas cítricas apresentaram maior riqueza e maior diversidade na comunidade de Coccinellidae. Como os coccinelídeos predadores foram mais numerosos, observa-se que, tanto a distribuição dentre as árvores do pomar quanto a distribuição sazonal, poderiam estar diretamente relacionadas à abundância de suas presas no pomar.

Os fatores climáticos que afetaram diretamente a comunidade de coccinelídeos foram a umidade e a precipitação, sendo que estes fatores estão relacionados à fenologia das plantas hospedeira de suas presas. $\mathrm{O}$ aumento no número de $H$. axyridis foi acompanhado pela redução no número das outras espécies, implicando uma sobreposição pela espécie invasora.

\section{REFERÊNCIAS}

Abreu, RLSD, C Sales-Campos, RE Hanada, FJD Vasconcellos \& JAD Freitas, 2002. Avaliação de danos por insetos em toras estocadas em indústrias madeireiras de Manaus, Amazonas, Brasil. Revista Árvore, 26: 789-796. DOI: https://doi.org/10.1590/s0100-67622002000600015.

Almeida, ML \& VB Silva, 2002. Primeiro registro de Harmonia axyridis (Pallas) (Coleoptera, Coccinellidae): um coccinelídeo originário da região Paleártica. Revista Brasileira de Zoologia, 19: 941-944.DOI: https://doi.org/10.1590/s010181752002000300031.

Arnett, R, M Thomas, P Skelley \& J Frank, 2002. American Beetles, Volume II: Polyphaga: Scarabaeoidea through Curculionoidea. $1^{\mathrm{a}}$ Ed., Boca Raton, CRC, Press. 880 p.

Cain, ML, WD Bowman \& SD Hacker, 2011. Ecologia, Porto Alegre, Artmed. 640 p.

Hammer, Ø, DAT Harper \& PD Ryan, 2013. PAST: Paleontological Statistics software package for education and data analysis. Available on: <http://https://folk.uio.no/ohammer/past>.

Koch, RL, 2003. The multicolored Asian lady beetle, Harmonia axyridis: a review of its biology, uses in biological control, and non-target impacts. Journal of Insect Science, 3: 1-16. DOI: https://doi.org/10.1093/jis/3.1.32.

Magurran, AE, 2003. Measuring Biological Diversity. $1^{\mathrm{a}}$ Ed., Malden, Blackwell Publishing. 264 p.
Matrangolo, WJR, I Cruz \& TMC Della Lúcia, 1997. Insetos fitófagos presentes em estilos-estigma e espigas de milho e avaliação de dano. Pesquisa Agropecuária Brasileira, 32: 773779 .

Michaud, JP, 2002. Invasion of the Florida Citrus Ecosystem by Harmonia axyridis (Coleoptera: Coccinellidae) and Asymmetric Competition with a Native Species, Cycloneda sanguinea. Environmental Entomology, 31: 5-827. DOI: https://doi.org/10.1603/0046-225x-31.5.827.

Milleo, J, JMT Souza, IF Barbola \& PE Husch, 2008. Harmonia axyridis em árvores frutíferas e impacto sobre outros coccinelídeos predadores. Pesquisa Agropecuária Brasileira, 43: 537-540. DOI: https://doi.org/10.1590/s0100204X2008000400013.

Moraes, RCB, ML. Haddad, SS Neto \& AEL Reyes, 2003. ANAFAU - Software para análise faunística. Available on: <http://www.lea.esalq.usp.br/softwares $>$.

Obrycki, JJ \& TJ Kring, 1998. Predaceous Coccinellidae in biological control. Annual Review of Entomology, 43: 295231. DOI: https://doi.org/10.1146/annurev.ento.43.1.295.

Obrycki, JJ, JD Hardwood, TJ Kring \& RJ O’Neil, 2009. Aphidophagy by Coccinellidae: application of biological control in agroecosystems. Biological Control, 51: 244-254. Doi: https://doi.org/10.1016/j.biocontrol.2009.05.009.

Oliveira, M, J Guerner-Moreira, MM Mesquita \& I Abreu, 2009. Important phytopathogenic airborne fungal spores in a rural area: Incidence of Botrytis cinerea and Oidium spp. Annals of Agricultural and Environmental Medicine, 2: 197-204.

Oliveira, AM, 1971. Observações sobre a influência de fatores climáticos nas populações de afídeos em batata. Pesquisa Agropecuária Brasileira, 6: 163-172.

Panizzi, ML \& JRP Parra, 2009. Bioecologia e nutrição de insetos: base para o manejo integrado de pragas, Brasília, Embrapa Informação Tecnológica. 1164 p.

Raven, PH, RF Evert \& SE Eichhorn, 2014. Biologia Vegetal, Rio de Janeiro, Guanabara Koogan. 876 p.

Roy, H \& E Wajnberg, 2008. Biological control to invasion: the ladybird Harmonia axyridis as a model species. BioControl, 53: $1-4$.

Santos-Cividanes, TE, FJ Cividanes \& BA Matos, 2007. Biologia de Psyllobora confluens alimentada com o fungo Erysiphe cichoracearums. Pesquisa Agropecuária Brasileira, 42: 1675-1679. DOI: https://doi.org/10.1590/s0100204X2007001200002.

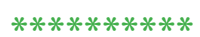

\section{Suggestion citation:}

Begha, BP, JMT Souza, CH Antunes \& J Milléo, 2019. Diversidade e sazonalidade de coccinelídeos (Coleoptera: Coccinellidae) em pomar no município de Ponta Grossa, Paraná. EntomoBrasilis, 12 (3): 108-112.

Available on: doi:10.12741/ebrasilis.v12i3.832

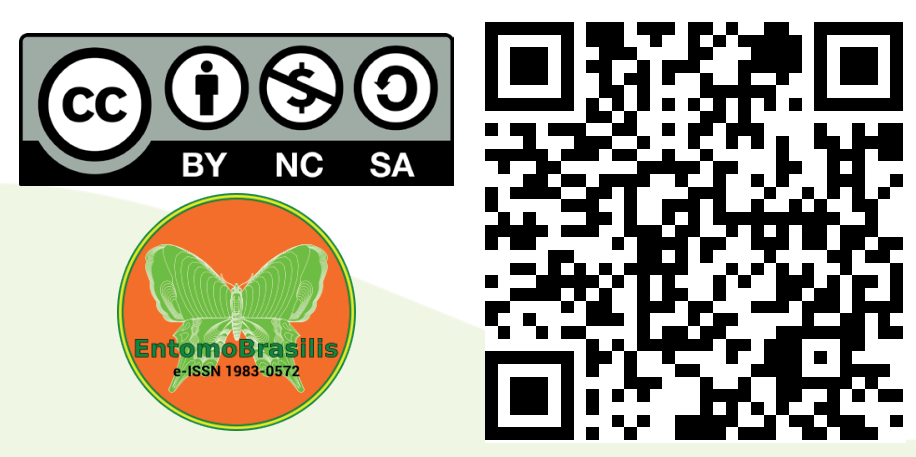

